\title{
From isoperimetric inequalities to heat kernels via symmetrisation
}

\author{
Gérard Besson \\ This paper is dedicated to M. Berger for his teaching.
}

\begin{abstract}
We briefly describe the series of work that led from the Paul LévyGromov isoperimetric inequality to the estimates on the heat kernel obtained by the technique of symmetrisation. An interpretation in terms of a spectral precompactness theorem is given at the end.
\end{abstract}

\section{Contents}

1. The classical isoperimetric inequality 28

2. The symmetrisations 29

3. The Faber-Krahn inequality 30

4. The Paul Lévy-Gromov isoperimetric inequality 33

5. Estimating eigenvalues 37

6. Estimating the heat kernel 39

7. Spectral precompactness theorems 42

8. Miscellaneous comments and questions 47

$\begin{array}{ll}\text { References } & 48\end{array}$

This text is a brief account of the series of work which led from isoperimetric inequalities to estimates on the heat kernel of a Riemannian manifold. The two basic ingredients are the Paul Lévy-Gromov isoperimetric inequality and the symmetrisation process which is a device allowing to translate geometric inequalities into analytic ones. Beyond estimating the heat kernel we shall describe a spectral version of Gromov's compactness theorem. This survey certainly cannot prevent from reading the deep results on isoperimetric inequalities described in the book [45]. The reader should also consult [15]. Further general references will be given

2000 Mathematics Subject Classification. Primary 54C21, 58J50; Secondary 53C20, 58J35. Key words and phrases. Differential geometry, algebraic geometry.

The author is partially supported by the European network E.D.G.E. HPRN-CT-2000-00101. 
in the sequel. The author apologizes for the restrictive choice made here and for possible omissions.

It is our pleasure to thank V. Bayle, D. Cordero and S. Gallot for many helpful conversations.

\section{The classical isoperimetric inequality}

The paradigmatic example of an isoperimetric inequality is the so-called classical one. It seems that it always has been known; indeed it is cited in Aristotle and is usually known as the solution to the Dido problem. In modern terms it is stated as follows: let $D$ be a domain in $\mathbb{R}^{2}$ with smooth boundary and $D^{*}$ be a ball, say centred at the origin, whose area is equal to the area of $D$; then,

$$
L(\partial D) \geq L\left(\partial D^{*}\right)
$$

where $L$ denotes the length of the boundary of the domains $D$ and $D^{*}$. Furthermore, the equality in the above inequality implies that $D$ is congruent (isometric) to $D^{*}$. Another way of stating it, without refereeing to the ball, is

$$
L^{2} \geq 4 \pi A
$$

where $L$ (resp. A) denotes again the length of the boundary of $D$ (resp. the area of $D$ ). In the first form, strictly speaking, it should rather be called an isovolumic inequality and its extension to higher dimension is obvious.

Various questions are raised by this inequality. One of them is the possible extension to domains whose boundary is not anymore smooth: what notion of "length of the boundary" can be used? answers can be found in the references $[\mathbf{2 7}],[\mathbf{2 2}]$ or $[\mathbf{6}]$ where notions such as the perimeter according to Cacciopoli and De Giorgi or the exterior Minkowski content are discussed. The best way to avoid considering such issues is to use the following form,

THEOREM 1.1 (Isoperimetric inequality in $\mathbb{R}^{n}$ ). Let $D$ be a domain in $\mathbb{R}^{n}$ and $D^{*}$ a ball which has the same volume. We denote by $D_{r}$ (resp. $\left.D_{r}^{*}\right)$ the $r$-tubular neighbourhood of $D$ (resp. $D^{*}$ ), then

$$
\operatorname{vol}\left(D_{r}\right) \geq \operatorname{vol}\left(D_{r}^{*}\right) .
$$

Although the inequality is natural, correct and complete proofs were only produced quite recently. Let us for example mention the proof, for $n=2$, by A. Hurwitz refined by $H$. Lebesgue (see $[\mathbf{4 9}],[\mathbf{5 0}]$ and [57]), at the undergraduate level, using a Fourier series expansion of the map from $\mathbf{S}^{1}$ to $\mathbf{C}$ defining the boundary of $D$. It does give an easy proof when the domain is simply-connected together with a stability result. We shall here give a proof using the mass transportation.

SKETCH OF PROOF. It relies on the idea of optimal mass transportation that was kindly communicated to us by D. Cordero (see [35]). It uses the following result proved by Y. Brenier [21] and extended by R. McCann [61],

TheOREM $1.2([\mathbf{6 1}])$. Let $\mu$ and $\nu$ be two probability measures on $\mathbb{R}^{n}$ and assume that $\mu$ is absolutely continuous with respect to the Lebesgue measure; then there exists a convex function $\phi$ such that $(\nabla \phi)_{*} \mu=\nu$. Furthermore, $\nabla \phi$ is unique $\mu$-ae.

Here $\nabla \phi$ is a vector field, thus a map from $\mathbb{R}^{n}$ to itself; then $(\nabla \phi)_{*} \mu$ is the pushed forward measure by this map. 
Now let $\mu=\frac{1}{\operatorname{vol}(D)} \mathbf{1}_{D}$ and $\nu=\frac{1}{\operatorname{vol}(B)} \mathbf{1}_{B}$, where $B$ is the unit ball. The above theorem asserts that there exists a convex function $\phi$ such that $(\nabla \phi)_{*} \mu=\nu$. The map $\nabla \phi$ sends $D$ into $B$ and thus, on $D,|\nabla \phi| \leq 1$. Furthermore, the equality between the two measures yields, by change of variables, a Monge-Ampère equation, i.e.,

$$
\operatorname{det}(\operatorname{hess} \phi(x))=\frac{\operatorname{vol}(B)}{\operatorname{vol}(D)}, \quad \forall x \in D
$$

Since $\phi$ is a convex function its hessian is non-negative and thus, for all $x \in D$,

$$
(\operatorname{det}(\operatorname{hess} \phi(x)))^{1 / n}=\left(\frac{\operatorname{vol}(B)}{\operatorname{vol}(D)}\right)^{1 / n} \leq \frac{1}{n}(\operatorname{trace}(\operatorname{hess} \phi(x)))=-\frac{1}{n} \Delta \phi(x)
$$

where $\Delta$ denotes the Laplacian as a positive operator. Then,

$$
\left(\frac{\operatorname{vol}(B)}{\operatorname{vol}(D)}\right)^{1 / n} \operatorname{vol}(D) \leq \frac{1}{n} \int_{D}(-\Delta \phi(x)) d x=\frac{1}{n} \int_{\partial D} \nabla \phi \cdot \vec{n}
$$

where $\vec{n}$ is the exterior unit normal to $\partial D$. We conclude by recalling that $|\nabla \phi(x)| \leq$ 1 for $x \in D$. This yields,

$$
\frac{1}{n} \operatorname{vol}_{n-1}(\partial D) \geq \operatorname{vol}(B)^{1 / n} \operatorname{vol}(D)^{1-1 / n}
$$

Remarks 1.3. (1) The article [21] contains interesting comments concerning the analogy between the result described in Theorem 1.2 and the polar decomposition of matrices. The analogy with the rearrangement of functions is also described.

(2) It is rather a sketch of the proof since in order to make the change of variables yielding the Monge-Ampère equation we would need to know that the map $\phi$ is at least $C^{2}$. This is obtained in this situation by the regularity results proved by L. Caffarelli (see $[\mathbf{2 3}],[\mathbf{2 4}]$ and $[\mathbf{2 5}]$ ).

(3) A similar proof gives the Brunn-Minkowski theorem. It is an easy exercise left to the reader. A very good survey of the inequalities related to the BrunnMinkowski theory is [43] (and the references herein).

(4) The extension to manifolds, even with constant curvature, seems completely open at this stage.

(5) A proof of the isoperimetric inequality by mass transportation is not new. Indeed, M. Gromov used the so-called Knothe transport. The map $\nabla \phi$ is replaced by a vector field whose Jacobian matrix is triangular and whose construction is explicit; the proof also extends to finite dimensional normed spaces. See the appendix I of $[62]$.

(6) In the article [35], the authors prove sharp Sobolev type inequalities using the mass transportation.

(7) A general reference for the theory of optimal transportation is [78].

\section{The symmetrisations}

The symmetrisations are natural processes used to replace a set by a more symmetric one; it has been invented by Jacob Steiner. When it is applied to the level sets of a function it allows to "rearrange" its values in order to produce a more symmetric function (invariant by some symmetries of the space). This technique is quite powerful and produce nice and sharp inequalities. The basic references are [71] and [6] in which a whole bunch of analytic inequalities are treated using various 
symmetrisations. Other good references for rearrangement are [46] and [22] (where several symmetrisations are discussed from a geometric point of view).

In this section we shall present in a very elementary way two symmetrisations that we shall need in the sequel.

2.1. Symmetrisation. Let $D$ be a domain in $\mathbb{R}^{n}$ and $V$ a $k$-dimensional affine subspace (where $k \leq n$ ), which we shall call a vertical subspace. For any $n-k$ dimensional affine subspace $\mathrm{H}$, orthogonal to $\mathrm{V}$, let us associate to $D \cap H$ a ball in $H$ of the same $n-k$-volume centred at the point $H \cap V$. When $H$ varies the union of these balls designed a domain which we shall also call $D^{*}$. Classically, the case $k=n-1$ is called the Steiner symmetrisation and the case $k=1$ is called the Schwarz symmetrisation (see $[\mathbf{7 1}]$ p.5 and $[\mathbf{2 2}]$ p.78). We shall be interested mainly in the two following cases: $k=n-1$ and $k=n$. The main feature of these elementary constructions is the

Proposition 2.1. In both cases,

1. $\operatorname{vol}(D)=\operatorname{vol}\left(D^{*}\right)$

2. $\operatorname{vol}_{n-1}(\partial D) \geq \operatorname{vol}_{n-1}\left(\partial D^{*}\right)$

Proof. For the $k=n$ symmetrisation, the equality (1) is in the definition of $D^{*}$ and the inequality (2) is the isoperimetric inequality.

For the Steiner and Schwarz symmetrisations, (1) is a corollary of Fubini's theorem. The inequality (2) can be proved by approximation (see [71] chapter 7 and $[\mathbf{2 2}]$ p. 79 for the spherical Steiner symmetrisation). In dimension 2, for example, if $V$ is a vertical line, one can approximate $D$ by trapezoids whose basis are horizontal. The inequality amounts then to compare the perimeter of such a trapezoid with a symmetric one (with respect to $V$ ).

\section{The Faber-Krahn inequality}

The Faber-Krahn inequality is the analytical translation of the isoperimetric inequality. It was conjecture by Lord Rayleigh in his book on the theory of sound $([\mathbf{7 7}]$ p. 339$)$ and proved independently by G. Faber $([\mathbf{3 8}])$ and E. Krahn $([\mathbf{5 5}])$. Let us consider a measurable function $u$ defined on a domain $D$ of $\mathbb{R}^{n}$. We assume furthermore that $u$ is positive on $D$ and vanishes on its boundary; it is sufficient to consider such functions for the following theorem. Let $D^{*}$ be a ball centred at the origin and having the same area as $D$.

Definition 3.1. If $\mathcal{E}$ denotes the domain in $\mathbb{R}^{n+1}$ below the graph of $u$ and above $D$, we define $\mathcal{E}^{*}$ to be the Schwarz-symmetrised domain. Its boundary consists of $D^{*}$ and the graph of a function from $D^{*}$ to $\mathbb{R}$ which we call $u^{*}$.

One can check that $u^{*}$ could also be defined as follows: let $D(\mu)=\{x \in D$ : $u(x) \geq \mu\}$, then, for $x \in D^{*}$, we define $u^{*}(x)=\sup \left\{\mu: x \in D(\mu)^{*}\right\}$ (see $[6]$ chapter II). The two functions $u$ and $u^{*}$ are thus seen to be equimeasurable, i.e the volumes of $D(\mu)$ and $D^{*}(\mu)$ (with an obvious definition) are equal. An important issue discussed in [6] is the regularity of $u^{*}$; it is, in particular, proved that if $u$ is a Lipschitz function so is $u^{*}$. In the sequel, we shall disregard these questions and the reader is referred to the above mentioned book.

Proposition 3.2. Let $D, D^{*}, u$ and $u^{*}$ be as above, then

1. If $u$ is a Lipschitz function so is $u^{*}$. 
2. For all $p \geq 0, \int_{D} u^{p}=\int_{D^{*}} u^{* p}$.

3. $\int_{D}|d u|^{2} \geq \int_{D^{*}}\left|d u^{*}\right|^{2}$.

Sketch OF PROOF of (3). We shall assume that all expressions below make sense. The key ingredient is the co-area formula (see [22] p. 103 or [39] section 3.2 ): let $h$ be a continuous function, then

$$
\int_{D} h(x) d x=\int_{0}^{\sup (u)}\left(\int_{\Gamma_{t}} h|d u|^{-1} d \sigma_{t}\right) d t
$$

where $\Gamma_{t}=u^{-1}(t)$ and $d \sigma_{t}$ is the volume element on $\Gamma_{t}$. Let us apply this formula to the set $\Omega_{t}=\{x \mid 0 \leq u(x)<t\} \subset D$ and $h \equiv 1$, this gives

$$
\operatorname{vol}\left(\Omega_{t}\right)=\int_{0}^{t}\left(\int_{\Gamma_{s}}|d u|^{-1} d \sigma_{s}\right) d s
$$

yielding $\frac{d}{d t}\left(\operatorname{vol}\left(\Omega_{t}\right)\right)=\int_{\Gamma_{t}}|d u|^{-1} d \sigma_{t}$. Now

$$
\left.\int_{D} u^{p}=\int_{0}^{\sup (u)} t^{p} \frac{d}{d t}\left(\operatorname{vol} \Omega_{t}\right)\right)=p \int_{0}^{\sup (u)} t^{p-1} \operatorname{vol}\left(\Omega_{t}\right) d t
$$

The equimeasurability of $u$ and $u^{*}$ and the last formula prove (2). We also get from the co-area formula,

$$
\int_{D}|d u|^{2}=\int_{0}^{\sup (u)}\left(\int_{\Gamma_{t}}|d u| d \sigma_{t}\right) d t
$$

and from the Cauchy-Schwarz inequality,

$$
\int_{\Gamma_{t}}|d u| d \sigma_{t} \geq\left(\int_{\Gamma_{t}} d \sigma_{t}\right)^{2} /\left(\int_{\Gamma_{t}}|d u|^{-1} d \sigma_{t}\right) .
$$

If we define $v_{n-1}(t)=\int_{\Gamma_{t}} d \sigma_{t}=\operatorname{vol}_{n-1}\left(\Gamma_{t}\right)$ and $v_{n}(t)=\operatorname{vol}\left(\Omega_{t}\right)$, we then get the inequality,

$$
\int_{\Gamma_{t}}|d u| d \sigma_{t} \geq \frac{\left(v_{n-1}(t)\right)^{2}}{v_{n}^{\prime}(t)} .
$$

For the function $u^{*}$ we get, with obvious notations,

$$
\int_{\Gamma_{t}}\left|d u^{*}\right| d \sigma_{t}=\frac{\left(v_{n-1}^{*}(t)\right)^{2}}{v_{n}^{*^{\prime}}(t)} .
$$

But, on the one hand by equimeasurability, we have $v_{n}(t)=v_{n}^{*}(t) \Rightarrow v_{n}^{\prime}(t)=v_{n}^{*^{\prime}}(t)$ and on the other hand the isoperimetric inequality gives $v_{n-1}(t) \geq v_{n-1}^{*}(t)$. Finally,

$$
\int_{\Gamma_{t}}|d u| d \sigma_{t} \geq \int_{\Gamma_{t}^{*}}\left|d u^{*}\right| d \sigma_{t} .
$$

The inequality (3) is then obtained by integration.

Let us give a slightly different point of view, which is more geometric.

Sketch of A SECOND PRoOf. (see [6] chapter II)

Let $\mathcal{G} \subset \mathbb{R}^{n+1}\left(\operatorname{resp} . \mathcal{G}^{*}\right)$ be the graph of $u$ (resp. $u^{*}$ ), the $n$-volume of $\mathcal{G}$ is given by,

$$
\operatorname{vol}_{n}(\mathcal{G})=\int_{D}\left(1+|d u|^{2}\right)^{1 / 2}
$$


Now the definition of $\mathcal{G}^{*}$ and the properties of the Schwarz symmetrisation give,

$$
\operatorname{vol}_{n}(\mathcal{G}) \geq \operatorname{vol}_{n}\left(\mathcal{G}^{*}\right) .
$$

We define $u_{\epsilon}=\sqrt{\epsilon} u$ for $\epsilon>0$. Clearly $(\sqrt{\epsilon} u)^{*}=\sqrt{\epsilon} u^{*}$, so that

$$
\int_{D}\left(1+\left|d u_{\epsilon}\right|^{2}\right)^{1 / 2} \geq \int_{D^{*}}\left(1+\left|d u_{\epsilon}^{*}\right|^{2}\right)^{1 / 2}
$$

Now letting $\epsilon$ go to 0 , expanding in $\epsilon$ and using the equality between the volumes of $D$ and $D^{*}$ give the inequality (3).

Remark 3.3. Once again let us emphasize that the co-area formula as used in the above "proof" needs some regularity on the function $u$. If $u$ is smooth, one can choose $t$ to be a regular value of $u$ thanks to Sard's theorem. One other possibility could be to approximate $u$ in a suitable topology by Morse functions with finitely many non degenerate critical points in the interior of $D$, an argument introduced by Th.Aubin (see [1] p. 40 or [14] lemma 10bis p. 519 and the appendix of [9]). This important issue is also discussed in [6].

We now proceed to the Faber-Krahn inequality. Let us denote by $\lambda_{1}(D)$ the first eigenvalue of the Laplace operator with Dirichlet boundary conditions on $D$ and $u$ a corresponding eigenfunction. We recall that $u$ has constant sign in the interior of $D$ (see $[\mathbf{3 7}]$ ); we thus can choose it to be positive. The key feature is the variational characterisation of the eigenvalues, also called the min-max principle, through the so-called Rayleigh quotient (see [6], [16], [9] and [37]). Precisely for $\lambda_{1}(D)$ one has,

$$
\lambda_{1}(D)=\inf \left\{\frac{\int_{D}|d u|^{2}}{\int_{D} u^{2}} \mid u \in C^{1}(D), u=0 \text { on } \partial D\right\} .
$$

Theorem 3.4 (The Faber-Krahn inequality). With the above notation,

$$
\lambda_{1}(D) \geq \lambda_{1}\left(D^{*}\right),
$$

with equality if and only if $D$ is a round ball.

Proof. The inequality is an immediate consequence of the Proposition 3.2. The equality case is more involved and the reader is referred to [6] or [71].

Remarks 3.5. (1) This inequality is the analytic translation of the isoperimetric inequality.

(2) The same proof also applies to some Sobolev constants as noticed by G. Talenti and Th. Aubin, see $[\mathbf{7 6}]$ and $[\mathbf{1}]$.

(3) In the article [36], dated from 1918, R. Courant proved that if $D \subset \mathbb{R}^{2}$ has smooth boundary and $D^{* *}$, is a ball with the same perimeter, then

$$
\lambda_{1}(D) \geq \lambda_{1}\left(D^{* *}\right) \text {. }
$$

Then, G. Faber (1923) and E. Krahn (1925) published independently the inequality which bears there names. One can easily check that $\lambda_{1}\left(D^{*}\right) \geq \lambda_{1}\left(D^{* *}\right)$ (it requires the monotonicity principle on Dirichlet eigenvalues described in [37] and [6]) which shows that Courant's inequality is weaker than the one by Faber and Krahn. Finally, let us mention that the proof of E. Krahn is simpler than the proof of G. Faber, in the spirit of the above sketch.

It seems that R. Courant suggested the problem to G. Faber giving the impulse in the gestation of this fundamental result. The article [36] was shown to us by M. Berger. 


\section{The Paul Lévy-Gromov isoperimetric inequality}

In this section we shall show how to extend the previous estimates on Riemannian manifolds. The core of it which is also the core of this text is the Paul Lévy-Gromov isoperimetric inequality. The basic reference is [45]. We shall use here the extension given in $[\mathbf{1 1}]$; the reader can also consult $[\mathbf{4 1}],[\mathbf{1 0}]$. For an n-dimensional closed Riemannian manifold $(M, g)$ and $0 \leq \beta \leq 1$, we let $h_{(M, g)}$ be the function defined by

$h_{(M, g)}(\beta)=\inf \left\{\frac{\operatorname{vol}_{n-1}(\partial \Omega)}{\operatorname{vol}(M)} \mid\right.$ for $\Omega \subset M$ with smooth boundary and $\left.\frac{\operatorname{vol}(\Omega)}{\operatorname{vol}(M)}=\beta\right\}$.

Definition 4.1. The function $h_{(M, g)}$ is called the isoperimetric profile of $\mathrm{M}$.

The isoperimetric profile captures the essence of the isoperimetry on $M$. Dividing by $\operatorname{vol}(M)$ amounts to normalising the Riemannian measure on $M$ to be a probability measure. This will facilitate comparisons between different Riemannian manifolds.

Example 4.2. On the $n$-sphere, endowed with its canonical metric, for a given value of $\beta$, the infimum in the definition of the isoperimetric profile is achieved for round balls (spherical caps) thanks to the isoperimetric inequality proved by E. Schmidt (see [74] and [22] p. 83 section 9.7). One can then compute the values of $h_{\left(S^{n}, \text { can }\right)}(\beta)$ for each $\beta$.

For example, when $n=2$, we have $h_{\left(S^{2} \text {,can }\right)}(\beta)=\sqrt{\beta(1-\beta)}$.

Remarks 4.3. (1) For a given value of $\beta$, different from 0 and 1 , a minimizing domain for the above infimum is easily seen to have constant mean curvature (where it is defined) and thus the computation of the function $h$, even on standard manifolds, requires the knowledge of constant mean curvature hypersurfaces. As a consequence there are very few explicit examples of isoperimetric profile. At this stage the isoperimetric profile of the 3-dimensional flat tori are not known.

(2) For the sake of simplicity we shall sometimes omit the reference to the metric $g$ in the isoperimetric profile when there is no ambiguity.

(3) The function $h_{M}$ satisfies obvious properties, such as the behaviour for $\beta$ going to 0 , the symmetry with respect to $1 / 2$, etc... The reader can consult $[\mathbf{9}] \mathrm{p}$. $85,[\mathbf{1 4}],[\mathbf{1 1}]$ or $[\mathbf{4 1}]$.

We now define

$$
R_{\text {min }}(x)=\inf \left\{\operatorname{Ricci}_{M}(u, u) \mid u \text { is a unit tangent vector at } x\right\},
$$

where $\operatorname{Ricci}_{M}$ denotes the Ricci curvature of $M$ as a field of bilinear forms and

$$
r_{\text {min }}=\inf \left\{R_{\text {min }}(x) \mid x \in M\right\} .
$$

We denote by $\operatorname{diam}(M)$ the diameter of $M$.

Theorem $4.4([\mathbf{1 1}])$. Let $(M, g)$ be an $n$-dimensional closed Riemannian manifold satisfying

$$
r_{\text {min }}(M) \operatorname{diam}(M)^{2} \geq \epsilon(n-1) \alpha^{2}
$$

for $\epsilon \in\{-1,0,1\}$ and $\alpha>0$. Then there exists a positive number $a(n, \epsilon, \alpha)$ such that, for all $\beta$ in $[0,1]$,

$$
h_{(M, g)}(\beta) \geq h_{\left(S^{n}(R), \text { can }\right)}(\beta),
$$


where $S^{n}(R)$ is the sphere of radius $R=\operatorname{diam}(M) / a(n, \epsilon, \alpha)$ with the induced (thus canonical) metric from $\mathbb{R}^{n+1}$. The number $a(n, \epsilon, \alpha)$ is explicit (see $[\mathbf{1 1}]$ for its value).

Remarks 4.5. (1) In [45] appendix C, M. Gromov states his result, namely when $r_{\text {min }}>0$. The above theorem is an improvement of the final step in the proof of Gromov's result, the general scheme of the proof being the same. Introducing the diameter allows to get rid of the positive curvature assumption. However we always compare to a round sphere.

(2) The inequality is sharp in the positive curvature case $(\epsilon=1)$.

Let us explain the different steps of the proof on a very simple case: $n=2$ and $M$ a convex surface in $\mathbb{R}^{2}$ whose curvature is not smaller than 1 . This is the original situation considered by Paul Lévy (see [58] Third part, chapter IV). This example contains all the ideas of the proof (see [42] Chapter IV section H).

Sketch OF PROOF. We proceed in three steps.

1st step: Let us fix $\beta$ different from 0 and 1 . The geometric measure theory (see [39]) asserts that there exists in $M$ an hypersurface $H$ (a curve in the case under consideration) bounding a domain $\Omega$, of relative volume $\beta$, such that $\frac{\operatorname{vol}_{n-1}(H)}{\operatorname{vol}(M)}=h(\beta)$. The existence is indeed easy since it boils down to proving a compactness theorem for certain rectifiable currents. More involved is the regularity theory in this situation. It is known that there may be singularities but they form a set of codimension at least 7 . In our (easy) case the curve $H$ is smooth and the mean curvature of $H$ is constant as can be seen by a standard variational technique. For the unit sphere the corresponding hypersurface $\partial \Omega_{r}^{*}$ is a circle around the north pole whose radius $r$ is computable in term of $\beta$.

2nd step: The main geometric ingredient is the Heintze-Karcher comparison theorem $([\mathbf{4 7}])$. More precisely $H$ is a two sided hypersurface and we choose a unit normal; furthermore any point in one side $(\Omega)$ or the other $\left(\Omega^{c}\right)$ is attained by the normal exponential to $H$. The volumes of $\Omega$ and $\Omega^{c}$ can be estimated if one has a control of the Jacobian of the normal exponential map, this is given in $[47]$; precisely, let $\eta$ be the (constant) mean curvature of $H$, then

$$
\operatorname{vol}(\Omega) \leq \operatorname{vol}_{n-1}(H) \int_{0}^{r}(\cos (t)-\eta \sin (t)) d t,
$$

where $r$ is the first point $t$ where $\cos (t)-\eta \sin (t)=0$, thus $\eta=\cot (r)$. The comparison theorem shows that if starting from $H$ we go in the normal direction in $\Omega$ up to distance $r$ we cover all of $\Omega$.

Now we can apply the same argument to $\Omega^{c}$. The mean curvature, with respect to the same normal is now $-\eta$, thus

$$
\operatorname{vol}\left(\Omega^{c}\right) \leq \operatorname{vol}_{n-1}(H) \int_{0}^{\pi-r}(\cos (t)+\eta \sin (t)) d t .
$$

The furthest point from $H$ in $\Omega^{c}$ is at most at distance diam $(M)-r$, but by Myers' theorem (see [42]) and since the curvature of $M$ has been assumed to be not greater than 1 in this case, one has $\operatorname{diam}(M) \leq \pi$; so if we replace $\operatorname{diam}(M)-r$ by $\pi-r$ we just increase the integral on the right hand side since the integrand is non-negative on the interval $[0, \pi-r]$. 
On the model space, the unit sphere, if $\Omega_{r}^{*}$ denotes the geodesic ball of radius $r$ around the north pole, one has

$$
\frac{\operatorname{vol}\left(\Omega_{r}^{*}\right)}{\operatorname{vol}_{n-1}\left(\partial \Omega_{r}^{*}\right)}=\int_{0}^{r}(\cos (t)-\eta \sin (t)) d t=\frac{1}{a(r)}
$$

indeed $\eta=\cot (r)$ is the mean curvature of $\partial \Omega_{r}^{*}$. In the same way,

$$
\frac{\operatorname{vol}\left(\Omega_{r}^{* c}\right)}{\operatorname{vol}_{n-1}\left(\partial \Omega_{r}^{* c}\right)}=\frac{1}{a(\pi-r)} \text {. }
$$

The conclusion is,

$$
\begin{aligned}
h_{M}(\beta)=\frac{\operatorname{vol}_{n-1}(H)}{\operatorname{vol}(M)} & \geq \max \left\{a(r) \operatorname{vol}(\Omega), a(\pi-r) \operatorname{vol}\left(\Omega^{c}\right)\right\} / \operatorname{vol}(M) \\
& \geq \max \{\beta a(r),(1-\beta) a(\pi-r)\} \\
& \geq \inf _{0 \leq t \leq \pi} \max \{\beta a(t),(1-\beta) a(\pi-t)\} .
\end{aligned}
$$

3rd step: The function $a(r)$ is explicitly computable (in this case) and is non decreasing. So the inf-max is achieved for $t_{0}$ such that

$$
\begin{aligned}
\beta a\left(t_{0}\right) & =(1-\beta) a\left(\pi-t_{0}\right) \\
\beta \frac{\operatorname{vol}_{n-1}\left(\partial \Omega_{t_{0}}^{*}\right)}{\operatorname{vol}\left(\Omega_{t_{0}}^{*}\right)} & =(1-\beta) \frac{\operatorname{vol}_{n-1}\left(\partial \Omega_{t_{0}}^{*}\right)}{\operatorname{vol}\left(\Omega_{t_{0}}^{* c}\right)}
\end{aligned}
$$

which means that $\Omega_{t_{0}}^{*}$ satisfies $\operatorname{vol}\left(\Omega_{t_{0}}^{*}\right)=\beta \operatorname{vol}\left(S^{2}\right)$. This proves the inequality,

$$
h_{(M, g)}(\beta) \geq h_{\left(S^{2}, \mathrm{can}\right)}(\beta) \text {. }
$$

Remark 4.6. In the positive curvature case $(\epsilon>0)$ the sketch of proof given above shows that there is an equality case. Indeed one can show that if $h_{(M, g)}(\beta)=$ $h_{\left(S^{n}, \text { can }\right)}(\beta)$ for one value of $\beta \in(0,1)$ then $(M, g)$ is isometric to $\left(S^{n}\right.$, can $)$. When the curvature is allowed to be non-positive, no such equality can be obtained.

Comments 4.7. One can try to extend the Theorem 4.4 in various directions. One of them is to try to understand more in depth the relationships between the curvature and $h$. This is the approach developed in the article by F. Morgan and D. Johnson in $[\mathbf{6 3}]$ and recently by V. Bayle in $[\mathbf{8}]$ where it is shown that $h_{(M, g)}$ satisfies a differential inequality in $\beta$ which yields a different proof of 4.4. More precisely, one can show the following

THEOREM $4.8([\mathbf{8}])$. Let $(M, g)$ be a closed $n$-dimensional Riemannian manifold satisfying $\operatorname{Ricci}(g) \geq(n-1) \delta g$, for $\delta \in \mathbb{R}$. Then the function $y=h_{(M, g)}^{n /(n-1)}$ satisfies the differential inequality

$$
y^{\prime \prime} \leq-n \delta y^{(2-n) / n},
$$

where the second derivative is taken in the sense of distributions.

The basic idea is to study the parallel deformations of the boundary of an optimal domain for a given value of $\beta$. The main issue is to deal with the singularities of $H$. This theorem gives a new proof of Paul Lévy-Gromov's isoperimetric inequality. In this proof the Heintze-Karcher comparison theorem is not used any more. One conclusion of all these works that should be emphasized is that rather than comparing our manifold with a sphere, we are indeed comparing it with an 
one dimensional model, consisting of an interval (or a union of intervals) with a relevant density; this was developed in details and with a series of examples in [40]. This is not that surprising since all the classical geometric comparison theorems (Bishop's, Bishop-Gromov's, etc... ) produce comparisons with constant curvature space which are rotationally symmetric, and thus, in spirit, one-dimensional. It is a question whether there exists a (reasonable) comparison geometry! with, sa! y the complex projective or hyperbolic space (for a survey of comparison geometry see $[66])$.

A second point of view is to try to replace $L^{\infty}$ norms on the Ricci curvature (lower bound of Ricci curvature as above) by $L^{p}$ norms. In this context this trend was initiated by S. Gallot in the paper [41]. It is worth citing the following result.

TheOREM $4.9([\mathbf{4 1}])$. Let $\alpha$ and $D$ be positive constants and $q \in(n,+\infty)$. Let $(M, g)$ be a closed n-dimensional Riemannian manifold whose diameter is bounded above by $D$ and whose Ricci curvature satisfies

$$
\frac{1}{\operatorname{vol}(M)} \int_{M}\left(\frac{r_{-}}{(n-1) \alpha^{2}}-1\right)_{+}^{q / 2} d v_{g} \leq \frac{1}{2}\left(e^{B(q) \alpha D}-1\right)^{-1},
$$

where $r_{-}(x)=\max \left(0,-R_{\min }(x)\right)$ and $(.)_{+}$denotes the positive part. Then, for every domain $\Omega \subset M$, we have

$$
\frac{\operatorname{vol}(\partial \Omega)}{\operatorname{vol}(M)} \geq \gamma(\alpha, D) \min \left(\frac{\operatorname{vol}(\Omega)}{\operatorname{vol}(M)}, \frac{\operatorname{vol}\left(\Omega^{c}\right)}{\operatorname{vol}(M)}\right)^{1-\frac{1}{q}} .
$$

The functions $B(q)$ and $\gamma(\alpha, D)>0$ are computed explicitly.

This result is quite optimal in the sense that the hypothesis cannot be relaxed too much as is shown in [41]. For example such estimates with $p=n$ are shown to be impossible.

Further interesting developments toward a geometry under integral bounds of curvature have been made, in particular in the articles [68] and [70] (and the references therein). Concerning the isoperimetric inequalities the most recent results in this direction are given in [2] by E. Aubry where the hypothesis on the diameter is dropped. More precisely, one gets a Myers's type theorem as follows.

TheOrem $4.10([\mathbf{2}])$. For any $q>n / 2$ there exist two explicitly computable positive constants $C(q, n)$ and $\alpha(q, n)$ such that the following is true:

1. If $0<R \leq 6 \pi$, then any $n$-dimensional complete Riemannian manifold $(M, g)$ such that there exists a point $x_{0} \in M$ satisfying

$$
\left\|\left(R_{\text {min }}-(n-1)\right)_{-}\right\|_{L^{q}\left(B\left(x_{0}, R\right)\right)} \leq \epsilon \leq \alpha(q, n),
$$

has its diameter bounded by $\pi\left(1+C(q, n) \epsilon^{\frac{q}{2 q-1}}\right)$. In particular, $M$ is compact.

2. If $R>6 \pi$, then the same conclusions hold under the more restrictive hypothesis

$$
R^{2}\left\|\left(R_{\text {min }}-(n-1)\right)_{-}\right\|_{L^{q}\left(B\left(x_{0}, R\right)\right)} \leq \epsilon \leq \alpha(q, n) .
$$

Here $B\left(x_{0}, R\right)$ denotes the geodesic ball centred at $x_{0}$ and of radius $R$.

A similar result appeared earlier in [69]; in [2] p. 134 E. Aubry suggests that the proof given in [69] is not complete; this is a point yet to be checked. Now, together with the first part of Theorem 4.9 this yields the isoperimetric inequality that appeared in the second statement in 4.9 with these (weaker) hypothesis. It is 
to be noticed that under the the above hypotheses the curvature is almost positive (in integral sense); however, the Ricci curvature may take very negative values on a small set. It is not yet a version of Paul Lévy-Gromov isoperimetric inequality under integral bounds on the Ricci curvature, but according to the author ([2] p.144) such a statement is attainable and is in progress. For a review of convergence and compactness theorems under integral bounds of the curvature see [66].

Finally another important approach is developed in the works by D. Bakry and M. Ledoux ([3] and also [4]). Here an abstract version of Lévy-Gromov's isoperimetric inequality is proved for an infinite dimensional diffusion generator involving comparison with Gaussian isoperimetric profile, which can be seen as a limit of profiles of suitably renormalised spheres whose dimensions tend to infinity $([7])$.

\section{Estimating eigenvalues}

As in section 3 we shall use the symmetrisation procedure to translate into analytic inequalities the isoperimetric inequality 4.4. The symmetrisation consists now to associate to a domain $\Omega$ in a closed manifold $M$ a geodesic ball $\Omega^{*}$ on the comparison n-sphere. More precisely, if $(M, g)$ satisfies $r_{\min }(M) \operatorname{diam}(M) \geq$ $\epsilon(n-1) \alpha^{2}$ as before, $\Omega^{*}$ is defined by,

$$
\frac{\operatorname{vol}(\Omega)}{\operatorname{vol}(M)}=\frac{\operatorname{vol}\left(\Omega^{*}\right)}{\operatorname{vol}\left(S^{n}(R)\right)}
$$

where $R$ is the explicit radius given in the Theorem 4.4. We shall now consider Riemannian Laplacian on $(\mathrm{M}, \mathrm{g})$. The first eigenvalue is always zero and by abuse of notation we shall call $\lambda_{1}(M)$ the first non-zero eigenvalue. It can be a multiple eigenvalue, and an eigenfunction corresponding to this eigenvalue changes sign; it indeed has exactly two nodal domains (see $[\mathbf{9}]$ ). The same procedure than the one described in section 3 leads to the following theorem:

THEOREM $5.1([\mathbf{1 1}])$. i) Under the hypotheses of Theorem 4.4, one has

$$
\lambda_{1}(M) \geq \lambda_{1}\left(S^{n}(R)\right)=n\left(\frac{a(n, \epsilon, \alpha)}{\operatorname{diam}(M)}\right)^{2} .
$$

ii) Under the same hypotheses, if in addition $r_{\min }(M) \geq(n-1)$, then

$$
\lambda_{1}(M) \geq n\left(\frac{\int_{0}^{\pi / 2}(\cos t)^{n-1} d t}{\int_{0}^{d / 2}(\cos t)^{n-1} d t}\right)^{2 / n} \geq n .
$$

Moreover, the equality in the first inequality in (5.1) implies that $(M, g)$ is isometric to $\left(S^{n}(R)\right.$, can $)$

The explicit expression that appears in ii) comes from the explicit value for $R$ (see [11]). The second part of this theorem is an improvement of the LichnérowiczObata result showing that with the assumption ii) one has $\lambda_{1}(M) \geq \lambda_{1}\left(S^{n}\right)=n$ and the equality case $([\mathbf{6 0}]$ and $[\mathbf{6 4}]$, see also $[\mathbf{9}])$.

Comments on the Proof. The only difference with the proof of the Theorem 3.4 is that a first eigenfunction takes positive and negative values. Let $u$ be such an eigenfunction, we write $u=u^{+}-u^{-}$where $u^{ \pm}=\sup \{ \pm u, 0\}$. We now symmetrise $u^{+}$into a function $\left(u^{+}\right)_{N}^{*}$ with respect to the north pole and $u^{-}$ 
into $\left(u^{-}\right)_{S}^{*}$ with respect to the south pole and define $u^{*}=\left(u^{+}\right)_{N}^{*}-\left(u^{-}\right)_{S}^{*}$. The Proposition 3.2 is still valid together with the fact that,

$$
\int_{M} u=\int_{S^{n}(R)} u^{*}=0 \text {. }
$$

The conclusion is obtained by applying the min-max principle for the first non zero eigenvalue on a closed Riemannian manifold, namely

$$
\lambda_{1}(M)=\inf \left\{\frac{\int_{M}|d u|^{2} d v_{g}}{\int_{M} u^{2} d v_{g}} \mid u \in C^{1}(M) \text { and } \int_{M} u d v_{g}=0\right\} .
$$

Once again this technique also allows to control the Sobolev constants as was first noticed independently by Th. Aubin and G. Talenti (see [1], [76], [41] and $[10])$.

Comments 5.2. The abstract approach developed by D. Bakry and Z. Qian (see [5]) extends to a large family of operators previous results obtained by P. Kröger ([56]) for the Laplacian on Riemannian manifolds. It yields estimate on the first non zero eigenvalue without going through symmetrisation and also improves the Lichnérowicz-Obata result. Furthermore it gives a sharp bound in the case when $r_{\text {min }} \geq 0$ improving a result due to J.Q. Zhong and H.C. Yang $([\mathbf{7 9}])$ which asserts that if $(M, g)$ is a compact Riemannian manifold with non-negative Ricci curvature, then $\lambda_{1}(M) \geq \frac{\pi^{2}}{\operatorname{diam}(M)^{2}}$. This last statement is optimal as can be seen by considering long thin tori. Theorem 5.1 does not provide a proof of this result. P. Kröger and later D. Bakry and Z. Qian put these two results in the same framework, which consists in comparing the first eigenvalue of the Riemannian manifold to those of some operators on an interval or a! union of intervals. It goes through interesting comparison theorems on the range and on the gradient of eigenfunctions (see also [59]). The method of D. Bakry and Z. Quian uses an abstract version of the notion of lower bound for the Ricci curvature and of the Bochner technique (see [10]). The fact that the model space is one-dimensional gives more flexibility and this point of view is pushed far away in these works as in [40]. At this stage, the approach of [56] extended by [5] is one of the most powerful for estimating the first eigenvalue; it is however not so easy to draw an explicit lower bound for $\lambda_{1}(M)$, and it would be interested to see if it can be adapted to integral bounds on the curvature as below. It remains also to interpret V. Bayle's results in the abstract framework of [5], if possible. For an overview the reader is refered to the introduction of [5].

Concerning this last point, i.e. integral bounds on the curvature, one can use the symmetrisation (see $[\mathbf{4 0}]$ and $[\mathbf{1 1}]$ ) to give a lower bound on $\lambda_{1}$. More precisely, combining the Theorem 5.2 in [69] and the Theorem 4.10 above we obtain the following result (see [2] section 4.3.4.).

Theorem $5.3([\mathbf{2}])$. Under the hypothesis of Theorem 4.10 above one gets,

$$
\lambda_{1}(M) \geq n\left(1-C^{\prime}(q, n)\right)
$$

where $C^{\prime}(q, n) \in(0,1)$ is an explicitly computable constant.

This is an almost sharp and explicit version of Lichnérowicz-Obata's result. 


\section{Estimating the heat kernel}

The heat kernel of a domain or a Riemannian manifold encodes the eigenfunctions and the spectrum of the space under consideration. We shall present here a brief account of estimates obtained in the spirit of the preceding section. Various other points of view concerning the heat equation can be developed, some of which are described in this issue. We shall concentrate here on the symmetrisation technique. General references for this section are $[\mathbf{1 6}]$ and $[\mathbf{9}]$.

6.1. The heat kernel. For the sake of simplicity let us consider the case of a closed Riemannian manifold $(M, g)$. The Laplacian $\Delta$ gives rise, by standard functional calculus, to another operator $e^{-t \Delta}$ which is infinitely regularizing. It thus has a kernel which we shall denote by $p_{M}(t ; x, y)$, where $t>0$ and $x$ and $y$ are points in $M$. This function is the fundamental solution of the heat equation, i.e. it satisfies the parabolic equation,

$$
\left\{\begin{aligned}
\left(\frac{\partial}{\partial t}+\Delta\right) p_{M}(t ; x, .) & =0 \\
\lim _{t \rightarrow 0^{+}} p_{M}(t ; x, y) d v_{g}(y) & =\delta_{x}
\end{aligned}\right.
$$

where $\delta_{x}$ is the Dirac measure at $x \in M$. The kernel has an (obvious) expression in terms of the eigenfunctions and eigenvalues of the Laplacian. Let $\lambda_{i}(M)$ be the $i$-th eigenvalue of the Laplacian of $(M, g)$, counted with multiplicity $(0 \leq i)$; we shall write $\lambda_{i}$ when there is no ambiguity. Let $\left(\phi_{i}\right)_{0 \leq i}$ be an orthonormal Hilbert basis of eigenfunctions of $L^{2}\left(M, d v_{g}\right)$; then

$$
p_{M}(t ; x, y)=\sum_{i=0}^{+\infty} e^{-\lambda_{i} t} \phi_{i}(x) \phi_{i}(y) .
$$

Recall that on any compact manifold the eigenvalues form an increasing sequence of numbers going to $+\infty$ and that their multiplicities are finite. The operator $e^{-t \Delta}$ is of the trace class and its trace is given by the following formulae:

$$
Z_{M}(t)=\operatorname{trace}\left(e^{-t \Delta}\right)=\int_{M} p_{M}(t ; x, x) d v_{g}(x)=\sum_{i=0}^{+\infty} e^{-t \lambda_{i}} .
$$

The semi-group property, i.e. $e^{-(t+s) \Delta}=e^{-t \Delta} e^{-s \Delta}$ is translated to the identity

$$
p_{M}(t+s ; x, y)=\int_{M} p_{M}(t ; x, z) p_{M}(s ; z, y) d v_{g}(z) .
$$

The important feature, which links the heat kernel to the geometry of $M$ more tightly is the Minakshisundaram-Pleijel asymptotic expansion (see [16] p. 204). Namely, there exist $C^{\infty}$ functions $u_{i}$ on $M \times M$ such that for any integer $q$ and for all $(x, y)$ in $M \times M$ near the diagonal,

$p_{M}(t ; x, y)=\frac{1}{(4 \pi t)^{n / 2}} e^{-r^{2}(x, y) / 4 t}\left(u_{0}(x, y)+t u_{1}(x, y)+\cdots+t^{q} u_{q}(x, y)+O\left(t^{q+1}\right)\right)$,

where $r(x, y)$ is the Riemannian distance between $x$ and $y$ in $M$, and $t \rightarrow 0$. It follows from the proof that this expansion can be differentiated term by term as many times as needed (see [16] p.213, [26] p.154 and [44]). The functions $u_{i}$ can be expressed as integrals of local quantities computable using the metric and its derivatives. 
6.2. Estimating the heat kernel. We shall explain how to symmetrise solutions of parabolic equations or equivalently families of functions; the idea is to compare a symmetrised solution to the solution of a symmetrised problem. The reader can refer to the book $[\mathbf{6}]$ chapter IV section 3 . In the context of Riemannian geometry it first appeared in $[\mathbf{1 3}]$, see also $[\mathbf{4 0}]$ and $[\mathbf{9}]$. Let us quote the comparison theorem proved in $[\mathbf{1 1}]$ using the method of $[\mathbf{1 3}]$.

Theorem 6.1 ([13] and $[\mathbf{1 1}])$. Let $(M, g)$ be an n-dimensional closed Riemannian manifold satisfying

$$
r_{m i n} \operatorname{diam}(M)^{2} \geq(n-1) \epsilon \alpha^{2}
$$

for some $\epsilon \in\{-1,0,1\}$ and some positive number $\alpha$. Then, for all $x \in M$ and $t>0$,

$$
\operatorname{vol}(M) p_{M}(t ; x, x) \leq \operatorname{vol}\left(S^{n}(R)\right) p_{S^{n}(R)}(t ; p, p)=Z_{S^{n}(R)}(t)=Z_{S^{n}(1)}\left(t / R^{2}\right),
$$

where $p$ is any point on the sphere $S^{n}(R)$ of radius $R=R(n, \epsilon, \alpha)$ (here $R$ is the same as in Theorem 4.4).

The last equality comes from the invariance of $p_{S^{n}(R)}$ on the two-point homogeneous manifold $S^{n}(R)$. An immediate and trivial corollary is the inequality

$$
Z_{M}(t) \leq Z_{S^{n}(R)}(t)=Z_{S^{n}(1)}\left(t / R^{2}\right) .
$$

Sketch of PRoOf, SEe $[\mathbf{6}],[\mathbf{1 3}],[\mathbf{9}],[\mathbf{1 0}]$ And $[\mathbf{4 0}]$. For the sake of simplicity let us put $V=\operatorname{vol}(M)$ and $V^{*}=\operatorname{vol}\left(S^{n}(R)\right)$. For $f \in C^{\infty}(M)$, a non-negative function on $M$, we define $D(s)=\{x \in M \mid f(x)>s\}$ and $a(s)=\frac{\operatorname{vol}(D(s))}{V}$ which is a non-increasing function. Let us define,

$$
\bar{f}(\beta)=\inf \{s, a(s)<\beta\},
$$

we then have $\bar{f}(a(s))=s$ for all regular values $s$. As in the proof of Proposition 3.2 , and with the same notations, at a regular value of $f$, we have

$$
a^{\prime}(s)=-\frac{1}{V} \int_{\partial D(s)}|d f|^{-1} d \sigma_{s} .
$$

We define now $F(\beta)=\int_{D(\bar{f}(\beta))} f(x) d v_{g}(x)$ and the co-area formula gives,

$$
F(\beta)=\int_{\bar{f}(\beta)}^{\bar{f}(0)}\left(\int_{\partial D(s)} f|d f|^{-1} d \sigma_{s}\right) d s=-V \int_{\bar{f}(\beta)}^{\bar{f}(0)} s a^{\prime}(s) d t=V \int_{0}^{\beta} \bar{f}(u) d u .
$$

The last equality is obtained by the change of variable $u=a(s)$. We deduce from Green's formula that

$$
\int_{D(\bar{f}(\beta))} \Delta_{M} f=\int_{\partial D(\bar{f}(\beta))}|d f| d \sigma_{s} \geq \operatorname{vol}_{n-1}(\partial D(\bar{f}(\beta)))^{2}\left(\int_{\partial D(\bar{f}(\beta))}|d f|^{-1} d \sigma_{s}\right)^{-1} .
$$

On the other hand,

$$
F^{\prime \prime}(\beta)=V \bar{f}^{\prime}(\beta)=V\left(a^{\prime}(\bar{f}(\beta))\right)^{-1} \leq 0
$$

since $a$ is non-increasing; we thus get,

$$
\int_{D(\bar{f}(\beta))} \Delta_{M} f \geq-\left(\frac{\operatorname{vol}_{n-1}(\partial D)}{V}\right)^{2} F^{\prime \prime}(\beta) .
$$


On $S^{n}(R)$, if $f^{*}$ is a radial function, then the level sets $D^{*}(s)$ are geodesic balls and $\left|d f^{*}\right|$ is constant on $\partial D^{*}(s)$. We then get equality in all the above inequalities, so that, with $F^{*}(\beta)=\int_{D^{*}\left(\bar{f}^{*}(\beta)\right)} f^{*}$, we have

$$
\int_{D^{*}\left(\bar{f}^{*}(\beta)\right)} \Delta_{S^{n}(R)} f^{*}=-\left(\frac{\operatorname{vol}_{n-1}\left(\partial D^{*}\right)}{V^{*}}\right)^{2} F^{*^{\prime \prime}}(\beta) .
$$

We now apply these computations to $f_{t}=p_{M}(t ; x,$.$) and f_{t}^{*}=p_{S^{n}(R)}(t ; p,$.$) where$ $p$ is any chosen point on the sphere. we define, as before $F(t, \beta)=\int_{\left\{f_{t}>\bar{f}_{t}(\beta)\right\}} f_{t}$ and, with a similar formula, $F^{*}$. With these choices the following lemma is proved in [6] (lemma 4.13, p.212),

LEMMA 6.2 .

$$
\frac{\partial F}{\partial t}=\int_{\left\{f_{t}>\bar{f}_{t}(\beta)\right\}} \frac{\partial}{\partial t} f_{t}=-\int_{\left\{f_{t}>\bar{f}_{t}(\beta)\right\}} \Delta_{M} f_{t}
$$

and a similar formula is true with $F^{*}$.

Combining the above lemma, the definition of the isoperimetric profile and the fact that $F^{\prime \prime}$ is non-positive, we get

$$
\begin{aligned}
\frac{\partial F}{\partial t} & \leq h_{M}^{2}(\beta) F^{\prime \prime}(\beta) \\
\frac{\partial F^{*}}{\partial t} & =h_{S^{n}(R)}^{2}(\beta) F^{*^{\prime \prime}}(\beta)
\end{aligned}
$$

Notice that the two functions $F$ and $F^{*}$ are defined on the same two-dimensional space $(0,+\infty) \times(0,1)$. It is now possible to compare them. We define $G=F-F^{*}$, the Paul Lévy-Gromov isoperimetric inequality implies,

$$
\frac{\partial G}{\partial t}-h_{S^{n}(R)}^{2}(\beta) \frac{\partial^{2} G}{\partial \beta^{2}} \leq 0 .
$$

The function $G$ satisfies $G(t, 0)=G(t, 1)=0$ for all $t>0$ and $\lim _{t \rightarrow 0^{+}} G(t, \beta)=0$ for all $\beta \in[0,1]$, then a parabolic maximum principle (see [72]) gives $F \leq F^{*}$, that is,

$$
V \int_{0}^{a} \bar{f}_{t}(\beta) d \beta \leq V^{*} \int_{0}^{a} \bar{f}_{t}^{*}(\beta) d \beta \quad \forall a \in[0,1] .
$$

The following lemma is proved in $[6] \mathrm{p} .174$,

LEMMA 6.3.

$$
V^{2} \int_{0}^{1} \bar{f}_{t}(\beta)^{2} d \beta \leq V^{* 2} \int_{0}^{1} \bar{f}_{t}^{*}(\beta)^{2} d \beta
$$

Now the co-area formula yields,

$$
V^{2} \int_{M}\left(p_{M}(t ; x, y)\right)^{2} d v_{g}(y) \leq V^{* 2} \int_{S^{n}(R)}\left(p_{S^{n}(R)}(t ; p, q)\right)^{2} d q
$$

and the semi-group property,

$$
V p_{M}(2 t ; x, x) \leq V^{*} p_{S^{n}(R)}(2 t ; p, p)
$$

here $p$ is again any point on the sphere. This is the desired inequality.

Comments 6.4. As for the Theorem 4.4, the above inequality is sharp for positive curvature $(\epsilon=1)$. If one allows non sharp results the same proof works using the Theorem 4.9 above. Precisely, 
Theorem $6.5([\mathbf{4 1}],[\mathbf{4 0}])$. Under the hypothesis of Theorem 4.9, one has

$$
\operatorname{vol}(M) p_{M}(t ; x, x) \leq p^{*}(b(q, \alpha, D) t),
$$

where $b(q, \alpha, D)$ is a computable function and $p^{*}$ is the heat kernel of a double cone computed at one vertex of this cone.

The double cone is just a convenient and geometric way of describing the onedimensional model considered (see [40] for the details). The non sharpness of this comparison is seen in the following upper bound,

$$
p^{*}(t) \leq C(q) t^{-q / 2} .
$$

Now, one can also obtain a similar result under the hypothesis of the Theorem 4.10 , that is the almost positive curvature condition. We leave to the reader to state such a theorem.

In [9] appendix A, an abstract point of view on symmetrisation is presented. More precisely, one considers two Hilbert spaces $\mathcal{H}$ and $\mathcal{K}$ and a cone $\mathcal{K}^{+} \subset \mathcal{K}$ satisfying few properties. A symmetrisation $S$ is a map from $\mathcal{H}$ onto $\mathcal{K}^{+}$fulfilling two conditions (see $[\mathbf{9}]$ p.163). Two examples are given:

1. the map $S(f)=|f|$ from the space of $L^{2}$ sections of a hermitian vector bundle on a manifold $M$ (for example complex-valued functions on $M$ ) to the cone of non-negative functions on $M$.

2. The second example is the symmetrisation of functions as used above, where the cone is the space of radial functions on the model.

The inequalities on the heat kernel are interpreted as dominations of operators (the two heat operators in the second example). A criterion for domination in term of the quadratic form associated to the operators is given and checked in both examples. In the first one, this is an extension of the so-called Beurling-Deny criterion for positivity, namely the positivity of the heat kernel is equivalent to the following inequality,

$$
\int_{M}|d| u||^{2} \leq \int_{M}|d u|^{2}
$$

where $u$ is a $C^{1}$-function on $M$. In other words the Dirichlet integral is reduced when taking the absolute value of the function. For the general case of a section it is related to the so-called Kato inequality as treated in [48]. In the second example the criterion is checked in details in $[\mathbf{9}]$ appendix A.

\section{Spectral precompactness theorems}

In this section we present an interpretation of the inequalities on the heat kernel in term of a precompactness theorem. The basic example is Gromov's precompactness theorem. Let

$$
\mathcal{M}_{n, k, D}=\{(M, g) \mid \operatorname{dim} M=n, \operatorname{Ricci}(g) \geq(n-1) k g, \operatorname{diam}(M, g) \leq D\} .
$$

M. Gromov defined a distance $d_{G H}$ on the family of Riemannian manifold, now called the Gromov-Hausdorff distance, and proved the following (see [45]):

Theorem $7.1([\mathbf{4 5}])$. The metric space $\left(\mathcal{M}_{n, k, D}, d_{G H}\right)$ is precompact.

We are going to define a distance between manifolds using the heat kernel. Before that, we need some consequences of Theorem 6.1. 
Corollary $7.2([\mathbf{1 2}])$. There exist explicit functions $A(n, k, D), B(n, k, D)$ and $C(n, k, D)$ such that, for all $(M, g) \in \mathcal{M}_{n, k, D}$,

1. $\forall j \geq 0, \quad \lambda_{j}(M) \geq A(n, k, D) j^{2 / n}$

2. $N_{M}(\lambda)=\sharp\left\{j \mid \lambda_{j}(M) \leq \lambda\right\} \leq 1+B(n, k, D) \lambda^{n / 2}$

3. $\forall x \in M, \forall \alpha \geq 0$,

$$
\sum_{j \geq 1} \lambda_{j}^{\alpha}(M) e^{-t \lambda_{j}(M)} \phi_{j}^{2}(x) \leq \frac{C(n, k, D)}{\operatorname{vol}(M)}(\alpha+1) t^{-(n+2 \alpha) / 2} .
$$

Remark 7.3. The first point is easy for a fixed Riemannian manifold thanks to Weyl's asymptotic formula (see [9]). What is important is the uniformity on the space $\mathcal{M}_{n, k, D}$. The third point is more interesting since it amounts to an estimate on the time derivative of "order" $\alpha$ of the heat kernel. Let us sketch the (easy) proof of this fact.

Sketch of PRoof of (3). For $x \in M$ let us define the measure $\mu_{x}$ on $\mathbb{R}_{+}$by $d \mu_{x}=\sum_{j \geq 1} \phi_{j}^{2}(x) \delta_{\lambda_{j}}$. Then

$$
\sum_{j \geq 1} \lambda_{j}^{\alpha} e^{-t \lambda_{j}} \phi_{j}^{2}(x)=\int_{\mathbb{R}} \lambda^{\alpha} e^{-t \lambda} d \mu_{x}(\lambda)
$$

An integration by parts yields,

$$
0 \leq \int_{\mathbb{R}} \lambda^{\alpha} e^{-t \lambda} d \mu_{x}(\lambda) \leq \int_{\mathbb{R}} \lambda^{\alpha-1}(\lambda t+\alpha) e^{-t \lambda} \mu_{x}([0, \lambda]) d \lambda
$$

but $\mu_{x}([0, \lambda])=\sum_{0<\lambda_{j} \leq \lambda} \phi_{j}^{2}(x)$, thus

$$
\begin{gathered}
\sum_{0<\lambda_{j} \leq \lambda} \phi_{j}^{2}(x) \leq e \sum_{0<\lambda_{j} \leq \lambda} e^{-\lambda_{j} / \lambda} \phi_{j}^{2}(x) \leq e \sup _{x \in M}\left(p_{M}\left(\frac{1}{\lambda} ; x, x\right)-\frac{1}{\operatorname{vol}(M)}\right) \\
\sum_{0<\lambda_{j} \leq \lambda} \phi_{j}^{2}(x) \leq \frac{e}{\operatorname{vol}(M)}\left(Z_{S^{n}(1)}\left(\frac{1}{\lambda R^{2}}-1\right)\right) \leq E(n, k, D) \frac{\lambda^{n / 2}}{\operatorname{vol}(M)}
\end{gathered}
$$

and we conclude by a simple integration.

7.1. Construction of some embeddings. We shall use the Hilbert space of sequences defined by

$$
l^{2}=\left\{\left(a_{j}\right)_{j \geq 1},\left.a_{i} \in \mathbb{C}\left|\sum_{j \geq 1}\right| a_{j}\right|^{2}<+\infty\right\} .
$$

For a Riemannian manifold $(M, g), \mathcal{A}$ will denote an orthonormal basis of $L^{2}\left(M, d v_{g}\right)$ consisting of eigenfunctions denoted by $\phi_{j}^{\mathcal{A}}$ (corresponding to the eigenvalue $\lambda_{j}$ ). There are plenty of possible choices, even if all eigenvalues are simple, since, in this case, one can choose $\phi_{j}^{\mathcal{A}}$ or $-\phi_{j}^{\mathcal{A}}$.

Definition 7.4. Let $(M, g)$ and $\mathcal{A}$ be as above. For any $t>0$, define the mapping $\Psi_{t}^{\mathcal{A}}$ by

$$
\begin{aligned}
\Psi_{t}^{\mathcal{A}}: M & \longrightarrow l^{2} \\
x & \longmapsto \sqrt{2}(4 \pi)^{n / 4} t^{(n+2) / 4}\left\{e^{-\lambda_{j} t / 2} \phi_{j}^{\mathcal{A}}(x)\right\}_{j \geq 1}
\end{aligned}
$$


The constants that appear are just useful normalisations. In spirit this is just the map $x \longmapsto p_{M}(t ; x,$.$) , but we need the target space to be independent of (M, g)$, that is why the eigenfunctions are used as coordinates in an abstract Hilbert space. We now can prove the following elementary theorem (see $[\mathbf{1 2}]$ ):

Theorem $7.5([\mathbf{1 2}])$. Let $(M, g)$ and $\mathcal{A}$ be fixed.

1. For all $t>0$, the mapping $\Psi_{t}^{\mathcal{A}}$ is an embedding of $M$ into $l^{2}$.

2. Let can be the canonical (Euclidean) metric in $l^{2}$ and $\operatorname{scal}(g)$ be the scalar curvature of $g$. Then

$$
\left(\Psi_{t}^{\mathcal{A}}\right)^{*}(\operatorname{can})=g+t / 3\left(\frac{1}{2} \operatorname{scal}(g) g-\operatorname{Ricci}(g)\right)+O\left(t^{2}\right),
$$

when $t \rightarrow 0$.

Remarks 7.6. It is clear that $\Psi_{t}^{\mathcal{A}}$ is equivariant with respect to the action of the isometry group of $M$ (if any) on $M$ and on $l^{2}$ by permuting the eigenfunctions in the same eigenspace. Consequently, when $(M, g)$ is homogeneous irreducible, one can check that $\left(\Psi_{t}^{\mathcal{A}}\right)^{*}(\operatorname{can})=C(t) g$, i.e. the embedding is isometric up to a constant. In general it is asymptotically isometric (up to a constant). The tensor that appears in the expansion of the pulled-back metric is geometrically significant however it is still open to draw nice geometric conclusion from this fact.

REMARKS ON THE PROOF. The proof is straightforward. The first part is a consequence of the fact that the eigenfunctions separate the points and of a similar property on their differentials. The second part consists in differentiating the Minakshisundaram-Pleijel asymptotic expansion. In fact one just have to differentiate the distance $r$ and the function $u_{0}$. Let us recall that $u_{0}$ is related to the volume element at $y$ computed in polar normal coordinates around $x$, and thus by differentiation the Ricci curvature appears. The metric comes from differentiating the distance and finally $u_{1}(x, x)=1 / 6 \operatorname{scal}(g)(x)$. The details are in [12].

Comments 7.7. The embedding is very similar to the case of minimal submanifolds of the sphere $S^{n-1}$ in which the coordinates of $\mathbb{R}^{n}$ are eigenfunctions when restricted to the submanifold (endowed with the induced metric). Such an embedding is also used in the study of compact harmonic manifolds (see Besse's nice embedding in [18]). The idea was indeed to mimic these cases.

7.2. Spectral distances. The idea is now to use the Hausdorff distance in $l^{2}$ to define a distance between manifolds. One minor difficulty is to deal with the ambiguity brought by the choices of the basis of $L^{2}\left(M, d v_{g}\right)$. This ambiguity is given by the product of the orthogonal groups of the eigenspaces; since they are finite dimensional, by compactness of $M$, the product group is compact. The set of such basis will be called $\mathfrak{B}(M)$. Let us call $E_{j}$ the eigenspace corresponding to $\lambda_{j}$ and $\mu_{j}$ the $j$-th eigenvalue but counted without multiplicity. For a finite dimensional space $E$ we define the distance between two orthonormal basis to be the Euclidean distance between the identity matrix and the transition matrix between these two basis and we call it $d_{E}$. Finally, for a basis $\mathcal{A}$ of $L^{2}\left(M, d v_{g}\right)$ of eigenfunctions we denote by $\mathcal{A}_{\mid E_{j}}$ the basis induced on $E_{j}$. 
Definition 7.8. $\quad$ 1. For any two orthonormal basis $\mathcal{A}$ and $\mathcal{B}$ of $L^{2}\left(M, d v_{g}\right)$ of eigenfunctions of the Laplacian, we set

$$
d(\mathcal{A}, \mathcal{B})^{2}=\sum_{j=1}^{+\infty} \mu_{j}^{-N} d_{E_{j}}\left(\mathcal{A}_{\mid E_{j}}, \mathcal{B}_{\mid E_{j}}\right)^{2}
$$

where $N>n / 2$.

2. We define the mapping $I_{t}^{\mathcal{A}}$ by

$$
\begin{aligned}
I_{t}^{\mathcal{A}}: M & \longrightarrow l^{2} \\
x & \longmapsto \sqrt{\operatorname{vol}(M)}\left\{e^{-\lambda_{j} t / 2} \phi_{j}^{\mathcal{A}}(x)\right\}_{j \geq 1}
\end{aligned}
$$

3. Denoting by $d_{H}$ the Hausdorff distance between compact sets of $l^{2}$, we define, for two Riemannian manifolds $(M, g)$ and $\left(M^{\prime}, g^{\prime}\right)$,

$$
d_{t}\left(M, M^{\prime}\right)=\max \left\{\sup _{\mathcal{A}} \inf _{\mathcal{A}^{\prime}} d_{H}\left(I_{t}^{\mathcal{A}}(M), I_{t}^{\mathcal{A}^{\prime}}\left(M^{\prime}\right)\right), \sup _{\mathcal{A}^{\prime}} \inf _{\mathcal{A}} d_{H}\left(I_{t}^{\mathcal{A}}(M), I_{t}^{\mathcal{A}^{\prime}}\left(M^{\prime}\right)\right)\right\}
$$

where $\mathcal{A} \in \mathfrak{B}(M)$ and $\mathcal{A}^{\prime} \in \mathfrak{B}\left(M^{\prime}\right)$ (in the notation $d_{t}\left(M, M^{\prime}\right)$ we omit the reference to the metrics).

The following proposition is easy to check,

Proposition 7.9. For any $t>0, d_{t}$ is a distance on the isometry classes of Riemannian manifolds. In particular $d_{t}\left(M, M^{\prime}\right)=0 \Rightarrow M$ and $M^{\prime}$ are isometric.

7.3. Precompactness. We can then prove

THEOREM $7.10([\mathbf{1 2}])$. The metric space $\left(\mathcal{M}_{n, k, D}, d_{t}\right)$ is precompact for all $t>0$.

This closes up this circle of ideas; indeed, the manifolds in the space $\mathcal{M}_{n, k, D}$, which is a precompact space when endowed with the Gromov-Hausdorff distance, satisfy an isoperimetric inequality, which implies estimates on the heat kernel, which in turn yields a spectral precompactness.

Proof. Let $h^{1}$ be the following Sobolev space of sequences:

$$
h^{1}=\left\{\left(a_{j}\right)_{j \geq 1},\left.a_{j} \in \mathbb{C}\left|\sum_{j \geq 1}\left(1+j^{2 / n}\right)\right| a_{j}\right|^{2}<+\infty\right\} .
$$

The Rellich theorem (see [73]) shows that the obvious injection $h^{1} \hookrightarrow l^{2}$ is compact, that is, the image of a bounded set in $h^{1}$ is relatively compact in $l^{2}$. Since the family of compact subsets of a compact metric space endowed with the Hausdorff distance is precompact, it suffices to show that the map $I_{t}^{\mathcal{A}}$ sends the manifolds of $\mathcal{M}_{n, k, D}$ into a bounded subset of $h^{1}$. This is given by the following estimates, for $x \in M \subset \mathcal{M}_{n, k, D}$ :

$$
\begin{aligned}
\left\|I_{t}^{\mathcal{A}}(x)\right\|_{h^{1}}^{2} & =\operatorname{vol}(M) \sum_{j \geq 1}\left(1+j^{n / 2}\right) e^{-\lambda_{j} t} \phi_{j}^{2}(x) \\
& \leq E(n, k, D) \operatorname{vol}(M) \sum_{j \geq 1}\left(1+\lambda_{j}\right) e^{-\lambda_{j} t} \phi_{j}^{2}(x) \\
& \leq F(n, k, D) t^{-n / 2}\left(1+t^{-1}\right) .
\end{aligned}
$$

Here we have used the Corollary 7.2 and the fact that $Z_{S^{n}(1)}(t)-1 \leq C(n) t^{-n / 2}$, for some constant $C(n)$. 
7.4. Some improvements. The first improvement can be made using Theorem 6.5. Corollary 7.2 above can be proved by means of the estimate of Theorem 6.5 , and then a precompactness result can be obtained. The only difference is that the heat kernel of the double cone satisfies now $p^{*}(t) \leq C(q) t^{-q / 2}$ for $t$ small, which is irrelevant in the above proof since $t$ is fixed. We thus define

$$
\begin{aligned}
\mathcal{M}^{*}(n, q, \alpha, D)=\{(M, g) \mid & \operatorname{dim} M=n, \operatorname{diam}(M) \leq D \text { and } \\
& \left.\frac{1}{\operatorname{vol}(M)} \int_{M}\left(\frac{r_{-}}{(n-1) \alpha^{2}}-1\right)_{+}^{q / 2} d v_{g} \leq \frac{1}{2}\left(e^{B(q) \alpha D}-1\right)^{-1}\right\} .
\end{aligned}
$$

THEOREM 7.11. The metric space $\left(\mathcal{M}^{*}(n, q, \alpha, D), d_{t}\right)$ is precompact for all $t>0$.

It is also probably possible to use the result $[\mathbf{2}]$ to get rid of the restriction on the diameter in some instances. A geometric precompactness theorem with integral norms on the Ricci curvature is proved by P. Petersen and G. Wei in [70].

The second improvement of the above technique is due to A. Kasue and $\mathrm{H}$. $\mathrm{Ku}-$ mura $([\mathbf{5 3}],[\mathbf{5 4}])$ and more recently to A. Kasue $([\mathbf{5 2}])$. The above embeddings are used in a more systematic way, precisely the space $C^{0}\left([0,+\infty], l^{2}\right)$ is considered together with the map

$$
I_{\mathcal{A}}[x](t)=\left(e^{-(t+1 / t) / 2} e^{-\lambda_{j} t / 2} \phi_{j}(x)\right)_{j \geq 0},
$$

for $x \in M$. Then the distance in $C^{0}\left([0,+\infty], l^{2}\right)$ is taken to be

$$
\Theta(\gamma, \sigma)=\sup \left\{\|\gamma(t)-\sigma(t)\|_{l^{2}} \mid t \in[0,+\infty]\right\} .
$$

An easy computation shows that, for $x$ and $x^{\prime}$ in $M$ :

$$
\Theta\left(I_{\mathcal{A}}[x], I_{\mathcal{A}}\left[x^{\prime}\right]\right)^{2}=\sup _{t>0} e^{(t+1 / t)}\left(p_{M}(t ; x, x)+p_{M}\left(t ; x^{\prime}, x^{\prime}\right)-2 p_{M}\left(t ; x, x^{\prime}\right)\right) .
$$

So, if one defines a new distance on $M$ by,

$$
d_{M}^{\mathrm{spec}}\left(x, x^{\prime}\right)^{2}=\sup _{t>0} e^{(t+1 / t)}\left(p_{M}(t ; x, x)+p_{M}\left(t ; x^{\prime}, x^{\prime}\right)-2 p_{M}\left(t ; x, x^{\prime}\right)\right),
$$

then $I_{\mathcal{A}}$ is a distance preserving mapping between $\left(M, d_{M}^{\text {spec }}\right)$ and $C^{0}\left([0,+\infty], l^{2}\right)$.

Then they consider in [54] a family $\mathcal{F}$ of compact connected Riemannian manifolds satisfying the following condition: there exist positive constants $\nu$ and $C_{U}$, such that for any $M \in \mathcal{F}$,

$$
p_{M}(t ; x, x) \leq \frac{C_{U}}{t^{\nu / 2}}, \quad \text { for } t \in(0,1], x \in M .
$$

This condition is implied by our assumptions on the Ricci curvature. The result is as follows.

Theorem $7.12([\mathbf{5 4}])$. The families $\mathcal{F}^{\text {spec }}=\left\{\left(M, d_{M}^{\text {spec }}\right)\right\}$ and $\mathcal{F}^{\text {geo }}=\{(M, g)\}$ are precompact for, respectively, the spectral distance in $C^{0}\left([0,+\infty], l^{2}\right)$ and the Gromov-Hausdorff distance.

Possible limits of manifolds in the above metric spaces have nice properties; they are shown to carry a Dirichlet form, a Radon measure, and a heat semi-group; the eigenvalues are shown to converge and the eigenfunctions too (in a certain sense, see [54] and the introduction of [52]). These are spaces on which one can do analysis; see [52] for more details on the limit spaces. 


\section{Miscellaneous comments and questions}

We have left aside other cases of interest, for example, the case of manifolds with boundary and the case of non-compact manifolds. A problem, which also would deserve a survey, is the question of stability of the eigenvalues. Briefly, on the $n$ sphere the first eigenvalue has multiplicity $n+1$ and one can ask to describe the manifolds with Ricci $\geq(n-1) g$ and such that the $n+1$ (or fewer) first eigenvalues are close to the minimal possible value given by Lichnérowicz-Obata's theorem. This is closely related to the deep works of T. Colding (see [33], [32] and [34]). Recent results have been obtained in [67], [2] and [17].

For the non-compact case we can add the following comments. Let $X$ be a compact manifold which carries an hyperbolic metric and let $\widetilde{X}$ be its universal cover. The heat kernel of the hyperbolic metric on $\widetilde{X}$ goes to zero uniformly on compact sets when $t$ goes to $+\infty$. However the measure $p_{\widetilde{X}}(t ; x,). d v_{g}$ converges, when $t$ goes to infinity, to the hyperbolic harmonic measure $\bar{p}_{\tilde{X}}(x, \theta) d \theta$, where $\bar{p}$ denotes here the hyperbolic Poisson kernel, $\theta$ is a point on $\partial \widetilde{X}=S^{n-1}$ and $d \theta$ is the canonical measure on $\partial \widetilde{X}=S^{n-1}$ (here one has to choose an origin on $\widetilde{X}$ ). In [20] we use a family of measures on $\partial \widetilde{X}$, indexed by the points in $\widetilde{X}$ and associated to a metric on $\widetilde{X}$ pulled-back from $X$, to embed $\widetilde{X}$ into an infinite dimensional sphere. We then show that! among all such embeddings (when the metric varies) the embedding using the hyperbolic harmonic measure is absolutely minimizing for the volume of a fundamental domain. This is exactly a non-compact version of Besse's nice embedding (see [20] for the details); we do prove that a compact locally harmonic and negatively curved manifold is locally symmetric which is the analogue of the result obtained by Z. I. Szabo ([75]) for compact simply-connected harmonic manifolds.

In the compact case no such geometric result has been proved yet. One possibility could be to use a variation of the above theme given in [12]. Let us consider the embedding of a Riemannian manifold $M$ into the unit sphere of $l^{2}$ defined by:

$$
K_{t}^{\mathcal{A}}(x)=\frac{1}{\left(\sum_{j \geq 1} e^{-\lambda_{j} t} \phi_{j}^{2}(x)\right)^{2}}\left\{e^{-\lambda_{j} t / 2} \phi_{j}(x)\right\}_{j \geq 1} ;
$$

then the pulled back metric satisfies

$$
\left(K_{t}^{\mathcal{A}}\right)^{*}(\operatorname{can})=1 / 2 t\left[g-\frac{t}{3} \operatorname{Ricci}(g)+O\left(t^{2}\right)\right] .
$$

QUESTION 1. Is $K_{t}^{\mathcal{A}}$ asymptotically minimal?

Another question is the study of limit spaces for the spectral distance. Although the results of $[\mathbf{5 2}],[\mathbf{5 3}]$ and $[\mathbf{5 4}]$ are very interesting, the link between these limits and those obtained in the works of J. Cheeger and T. Colding (see [28], [29], $[\mathbf{3 0}],[\mathbf{3 1}])$ is not done. Let us call them the geometric limits. One result given by J. Cheeger and T. Colding (see [29]) is that when the limit of a sequence of compact Riemannian manifolds $M_{n}$ with Ricci curvature bounded below (by the same constant) is a compact manifold $M_{\infty}$, then, for $n$ large, $M_{n}$ is diffeomorphic to $M_{\infty}$. It would be interesting to give another proof of this theorem.

QUESTION 2. Can geometric limits be embedded in $l^{2}$, by a reasonable version of the heat kernel, onto the spectral limits? 
The embeddings presented in this text are related to the spectrum of the Laplacian, in particular when two Riemannian manifolds $(M, g)$ and $(N, h)$ are isospectral the embeddings satisfy

$$
Z_{M}(t)=\frac{1}{\operatorname{vol}(M)} \int_{M}\left\|I_{t}^{\mathcal{A}}(x)\right\|_{l^{2}}^{2} d v_{g}(x)=\frac{1}{\operatorname{vol}(N)} \int_{N}\left\|I_{t}^{\mathcal{B}}(x)\right\|_{l^{2}}^{2} d v_{h}(x)=Z_{N}(t),
$$

for all $t>0$. Let us recall that the spectrum of the Laplacian determines the volume of the manifold. This means that the image manifolds in $l^{2}$ have, for all $t>0$, the same moment of inertia.

QUESTION 3. Can this technique say something about the isospectral problem?

Another important question is to know what can be obtained by using other operators. Possible candidates are the Yamabe operator (see [19]) and the Dirac operator (see [51]). One can also study perturbed heat kernel, i.e. instead of defining the kernel by the parabolic equation considered above one could use the equation associated to an operator of the type:

$$
\frac{\partial}{\partial t}+\Delta_{g(t)}
$$

the difference being here that the metric varies with $t$, for example $g(t)$ could be the variation of metrics obtained from the Ricci flow. This kind of situation appears in G. Perel'man's recent works $([\mathbf{6 5}])$. A wild question can be:

Question 4. Can one see the Ricci flow as a family of submanifolds of $l^{2}$ ?

\section{References}

[1] Th. Aubin. Non linear analysis on Manifolds. Monge-Ampère equations, volume 252 of Grundlehren der mathematischen Wissenschaften. Springer-Verlag, 1982.

[2] E. Aubry. Variétés de coubure de Ricci minorée : inégalités géométriques optimales et stabilité des variétés extrémales. PhD thesis, Université Joseph Fourier (Grenoble I), December 2003.

[3] D. BAKRY and M. Ledoux. Lévy-Gromov's isoperimetric inequality for an infinite dimensional diffusion generator. Invent. math., 123:259-281, 1996.

[4] D. BAKRY and M. Ledoux. Sobolev inequalities and myers's diameter theorem for an abstract Markov generator. Duke Math. Journal, 85(1):253-270, 1996.

[5] D. BAKRY and Z. Quian. Some new results on eigenvectors via dimension, diameter and Ricci curvature. Advances in Math., 155:98-153, 2000.

[6] C. BANDLE. Isoperimetric Inequality and Applications, volume 7 of Monograph and Studies in Math. Pitman Press, 1980.

[7] F. Barthe. Log-concave and spherical models in isoperimetry. Geom. and Funct. Anal., 12:32-55, 2002.

[8] V. BAYle. A differential inequality for the isoperimetric profile. International Math. Res. Not., 7:311-342, 2004.

[9] P. BÉrard. Spectral Geometry: Direct and Inverse Problems, volume 1207 of Lecture Notes in Mathematics. Springer-Verlag, 1986.

[10] P. BÉRARD. From vanishing theorems to estimating theorems: the Bochner technique revisited. Bulletin of the A.M.S., 19(2):371-406, 1988.

[11] P. BÉrard, G. Besson, and S. Gallot. Sur une inégalité isopérimétrique qui généralise celle de Paul Lévy-Gromov. Invent. Math., 80:295-308, 1985.

[12] P. Bérard, G. Besson, and S. Gallot. Embedding Riemannian manifolds by their heat kernel. G.A.F.A., 4(4):373-398, 1994.

[13] P. BÉRARD and S. GALLOT. Inégalités isopérimétriques pour l'équation de la chaleur et applications à l'estimation de quelques invariants. In Séminaire Goulaouic-Meyer-Schwartz, Éxposé no. 15. École Polytechnique, 1983-84. 
[14] P. BÉrard and D. MEyer. Inégalités isopérimétriques et applications. Ann. Sci. E.N.S. Paris, 15:513-542, 1982.

[15] M. Berger. Riemannian geometry during the second half of the century. Number 17 in University Lecture Series. American Mathematical Society, 2000.

[16] M. Berger, P. Gauduchon, and E. Mazet. Le spectre d'une variété riemannienne, volume 194 of Lecture notes in Math. Springer-Verlag, 1971.

[17] J. Bertrand. Pincement spectral en courbure positive. PhD thesis, Université Paris-Sud, Orsay, Octobre 2003.

[18] A.L. Besse. Manifolds all of whose geodesics are closed. Number 93 in Ergebnisse der mathematik und ihrer grenzgebiete. Springer-Verlag, 1978.

[19] A.L. Besse. Einstein Manifolds. Number 10 in Ergebnisse der mathematik und ihrer grenzgebiete. 3 folge. Springer-Verlag, 1987.

[20] G. Besson, G. Courtois, and S. Gallot. Volume et entropie minimales des variétés localement symétriques. G.A.F.A., 5(5):731-799, 1995.

[21] Y. Brenier. Polar factorization and monotone rearrangement of vector-valued functions. Comm. Pure Appl. Math., 44:375-417, 1991.

[22] Yu.D. Burago and V.A. Zalgaller. Geometric Inequalities, volume 194 of Grundlehren der Math. Wiss. Springer-Verlag, 1985.

[23] L. Caffarelli. Boundary regularity of maps with convex potentials. Comm. Pure Appl. Math., 45(9):1141-1151, 1992.

[24] L. CAffarelli. The regularity of mappings with a convex potential. J. Amer. Math. Soc., $5(1): 99-104,1992$

[25] L. Caffarelli. Boundary regularity of maps with convex potentials ii. Ann of Math.(2), 144(3):453-496, 1996.

[26] I. Chavel. Eigenvalues in Riemannian geometry. With a chapter by B. Randol. With an appendix by J. Dodziuk. Number 115 in Pure and Applied Mathematics. Inc, 1984.

[27] I. Chavel. Isoperimetric inequalities. Number 145. Cambridge University Press, 2001.

[28] J. Cheeger and T. Colding. Lower bounds on Ricci curvature and the almost rigidity of warped products. Ann. of Math, 144:189-237, 1996.

[29] J. Cheeger and T. Colding. On the structure of spaces with curvature bounded below I. Journal of Diff. Geom., 46:406-480, 1997.

[30] J. Cheeger and T. Colding. On the structure of spaces with curvature bounded below II. Journal of Diff. Geom., 54:13-35, 2000.

[31] J. Cheeger and T. Colding. On the structure of spaces with curvature bounded below III. Journal of Diff. Geom., 54:37-74, 2000.

[32] T. Colding. Large manifolds with positive Ricci curvature. Invent. Math., 124:193-214, 1996.

[33] T. Colding. Shape of manifolds with positive Ricci curvature. Invent. Math., 124:175-191, 1996.

[34] T. Colding. Ricci curvature and volume convergence. Annals of Math, 145:477-501, 1997.

[35] D. Cordero-Erausquin, B. Nazaret, and C. Villani. A Mass-Transportation approach to sharp Sobolev and Gaglardo-Nirenberg inequalities. Advances in Math., 2004. to appear.

[36] R. Courant. Beweis des satzes daßvon allen homogenen Membranen gegebenen Umfanges und gegebener Spannung die Kreisförmige den tiefsten Grundton besitzt. Math. Zeitschrift, 1:321-328, 1928.

[37] R. Courant and D. Hilbert. Methods of mathematical physics, volume I and II. WileyInterscience, I 1953, II 1962.

[38] G. FABer. Beweis, dass unter allen homogenen Membranen von gleicher Fläche und gleicher Spannung die Kreisförmige des tiefsten Grundton gibt. Sitzungsber. Bayer. Akad. Wiss., Math. Phys. München, pages 169-172, 1923.

[39] H. Federer. Geometric Measure Theory, volume 153 of Grundlehren der mathematischen Wissenschaften. Springer-Verlag, 1969.

[40] S. Gallot. Inégalités isopérimétriques et analytiques sur les variétés riemanniennes. In Ida Cattaneo Gasparini, editor, On the geometry of differentiable manifolds, Roma, june 23-27, 1986, volume 163-164 of Astérisque, pages 31-91. Société Mathématique de France, 1988.

[41] S. GALlot. Isoperimetric inequalities based on integral norms of the Ricci curvature. In Colloque Paul Lévy sur les processus stochastiques, volume 157-158 of Astérisque, pages 191-216. Société Mathématique de France, 1988. 
[42] S. Gallot, J. Lafontaine, and D. Hulin. Riemannian Geometry. Universitext. SpringerVerlag, 2nd edition, 1990.

[43] R. J. Gardner. The Brunn-Minkowski inequality. Bull. Amer. Math. Soc., 39(3):355-405, 2002.

[44] P.B. Gilkey. Invariance theory, heat equation and the Atiyah-Singer index theory. Studies in Advanced Mathematics. CRC Press, 2nd edition, 1995.

[45] M. Gromov. Metric structures for Riemannian and non-Riemannian spaces. With appendices by M. Katz, P. Pansu and S. Semmes, volume 152 of Progress in Mathematics. Birkhäuser, 2nd edition, 1999.

[46] G. Hardy, J. Littlewood, and G. Polya. Inequalities. Cambridge University Press, Cambridge, 1967.

[47] E. Heintze and H. Karcher. A general comparison theorem with applications to volume estimates for submanifolds. Ann. Sci. Ec. Norm. Sup., 11:451-470, 1978.

[48] H. Hess, R. Schrader, and D. Uhlenbrock. Kato's inequality and the spectral distribution of Laplacians on compact Riemannian manifolds. Journal of Diff. Geom., 15:27-37, 1980.

[49] A. Hurwitz. Sur le problème des isopérimètres. C. R. Acad. Sci. Paris Sér. I Math., 132:401403, 1901.

[50] A. Hurwitz. Sur quelques applications géométriques des séries de Fourier. Ann. Sci. École Norm. Sup. (3), 19:357-408, 1902.

[51] B.H. Lawson J. and M-L. Michelson. Spin Geometry. Number 38 in Princeton mathematical series. Princeton University Press, 1989.

[52] A. Kasue. Convergence of Riemannian manifolds and Laplace operators I. Ann. Inst. Fourier, Grenoble, 52(4):1219-1257, 2002.

[53] A. Kasue and H. Kumura. Spectral convergence of Riemannian manifolds. Tôhoku Math. Journal, 46:147-179, 1994.

[54] A. Kasue and H. Kumura. Spectral convergence of Riemannian manifolds II. Tôhoku Math. Journal, 48:71-120, 1996.

[55] E. Krahn. Über eine von rayleigh formulierte Minimaleigenshaft des Kreises. Math. Annalen, 94:97-100, 1925.

[56] P. KröGer. On the spectral gap for compact manifolds. J. Diff. Geom., 36:315-330, 1992.

[57] H. Lebesgue. Leçons sur les séries trigonométriques. Gauthier-Villars, Paris, 1906.

[58] P. LÉvy. Problèmes concrets d'analyse fonctionnelle. Gauthier-Villars, Paris, 1951.

[59] P. Li and S.T. YAU. Estimates of eigenvalues of a compact riemannian manifold. volume 36 of Proc. Sympos. Pure Math., pages 205-239. American Math. Soc., Providence, R.I., 1980.

[60] A. Lichnérowicz. Géométrie des groupes de transformations, volume 3 of Travaux et recherches mathématiques. Dunod, 1958.

[61] R. MCCAnn. Existence and uniqueness of monotone measure-preserving maps. Duke Math. Journal, 80:309-323, 1995.

[62] V.D. Milman and G. Schechtman. Asymptotic Theory of Finite Dimensional Normed Spaces, volume 1200 of Lecture Notes in Math. Springer-Verlag, 1980.

[63] F. Morgan and D.L. Johnson. Some sharp isoperimetric theorems for Riemannian manifolds. Indiana Univ. Math. J., 49(3):1017-1041, 2000.

[64] M. ОватA. Certain conditions for a Riemannian manifold to be isometric with a sphere. $J$. Math. Soc. Japan, 14:333-340, 1962.

[65] G. Perel'man. The entropy formula for the Ricci flow and its geometric applications. ArXiv: Math. DG/ 0211159v1, Nov. 112002.

[66] P. Petersen. Convergence theorems in Riemannian geometry. In Karsten Grove and Peter Petersen, editors, Comparison geometry, volume 30 of M.R.S.I. Publications, pages 167-202. Cambridge University Press, 1997.

[67] P. Petersen. On eigenvalue pinching in positive Ricci curvature. Invent. Math., 138:1-21, 1999.

[68] P. Petersen, S. Shteingold, and G. Wei. Comparison geometry with integral curvature bounds. Geom. and Funct. Anal., 7:1011-1030, 1997.

[69] P. Petersen and C. Sprouse. Integral curvature bounds, distance estimates and applications. J. Diff. Geom., 50:269-298, 1998.

[70] P. Petersen and G. Wei. Relative volume comparison with integral curvature bounds. Geom. and Funct. Anal., 7:1031-1045, 1997. 
[71] G. Pólya and G. Szegö. Isoperimetric inequalities in mathematical physics. Number 27 in Annals of Mathematics Studies. Princeton University Press, 2nd edition, 1966.

[72] M.H. Protter and H.F. Weinberger. Maximum principles in differential equations. Prentice-Hall, Englewood Cliffs, N.J., 1967.

[73] M. ReED and B. Simon. Methods of modern mathematical physics, volume I-IV. Academic Press, 1975.

[74] E. Schmidt. Beweis der isoperimetrischen Eigenshaft der Kugel im hyperbolischen und sphärischen Raum jeder Dimensionenzahl. Math. Zeitschrift, 49:1-109, 1943.

[75] Z.I. Szabo. The Lichnérowicz conjecture on harmonic manifolds. Journal of Diff. Geom., $31: 1-28,1990$.

[76] G. Talenti. Elliptic equations and rearrangements. Ann. Scuola Norm. Sup. Pisa, 3:697-718, 1976.

[77] J. W. Strutt (Lord Rayleigh). The Theory of Sound. reprinted by Dover in 1945, 1894.

[78] C. Villani. Topics in Optimal Transportation, volume 58 of Graduate Studies in Mathematics. American Mathematical Society, Providence R.I., 2003.

[79] J.Q. ZHong and H.C. YANG. On the estimate of the first eigenvalue of a compact Riemannian manifold. Scientia Sinica Ser. A, 27(12):1265-1273, 1984.

Institut Fourier, U.M.R. 5582 Du C.N.R.S., Université De Grenoble I, B.P. 74, 38402 Saint Martin d'Hères Cedex, France

E-mail address: G.Besson@ujf-grenoble.fr 